\title{
Politique
}

\section{Index des articles publiés dans la revue Politique}

Numéro 16, automne 1989

Les États-Unis

URI : https://id.erudit.org/iderudit/040645ar

DOI : https://doi.org/10.7202/040645ar

Aller au sommaire du numéro

Éditeur(s)

Société québécoise de science politique

ISSN

0711-608X (imprimé)

1918-6584 (numérique)

Découvrir la revue

Citer ce document

(1989). Index des articles publiés dans la revue Politique. Politique, (16),

157-172. https://doi.org/10.7202/040645ar d'utilisation que vous pouvez consulter en ligne.

https://apropos.erudit.org/fr/usagers/politique-dutilisation/ 


\section{Index des articles publiés dans la revue Politique nos 1-15 (par ordre alphabétique)}

ANDERSON, Frances, BOISMENU, Gérard et DUCATENZEILER, Graciela, «L'aide directe fédérale à l'innovation industrielle», Revue Politique, Innovations et politiques technologiques, $\mathrm{n}^{\circ} 8,1985, \mathrm{pp} .45-76$.

ANDREW, Caroline, «Les femmes et la consommation collective: les enjeux de l'engagement politique», Revue Politique, Femmes et pouvoir, $\mathrm{n}^{\circ}$ 5, 1984, pp. 107-122.

BAUER, Julien, «Résolution des conflits et crise de décision», Revue Politique, Crise de décision, $\mathrm{n}^{\circ} 13,1988$, pp. 5-36.

BERGERON, Gérard, «De la tour d'ivoire à la place publique», Revue Politique, Les intellectuels et le pouvoir, $\mathrm{n}^{\circ} 1$, 1982, pp. 9-18.

BERNARD, André, «Les «experts» et le pouvoir», Revue Politique, Les intellectuels et le pouvoir, $\mathrm{n}^{\circ} 1,1982, \mathrm{pp} .47-$ 61.

BLAIS, André et DION, Stéphane, «Trop d'État? Un baromètre de l'opinion», Revue Politique, L'État privé, n 11, 1987, pp. 43-72. 
BLAIS, André et CRETE, Jean, «La clientèle péquiste en 1985: caractéristiques et Évolution», Revue Politique, Opinions et votes, $\mathrm{n}^{\circ} 10,1986, \mathrm{pp}$. 5-29.

BLAIS, André et CRETE, Jean, "La presse et la politique municipale dans deux villes du Québec», Revue Politique, Les médias et les pouvoirs, $n^{\circ} 2,1982$, pp. 41-67.

BOILY, Nicole, «Les femmes en politique, encore une exception», entrevue réalisée par Carolle Simard et Denis Monière, Revue Politique, Femmes et pouvoir, $\mathrm{n}^{\circ} 5,1984$, pp. 7 25.

BOISMENU, Gérard, DUCATENZEILER, Graciela, et ANDERSON, Frances, «L'aide directe fédérale à l'innovation industrielle», Revue Politique, Innovations et politiques technologiques, $n^{\circ} 8,1985$, pp. 45-76.

BOUCHER, Michel et CARTER, Richard, «Dynamique politique et déréglementation», Revue Politique, L'État privé, $\mathrm{n}^{\circ} 11$, 1987, pp. 107-125.

BOUCHER, Michel, «Le référendum de mai 1980: présentation de quelques résultats statistiques», Revue Politique, $L a$ réforme des institutions politiques, $\mathrm{n}^{\circ} 6,1984, \mathrm{pp}$. 103124.

CAMbrosio, Alberto, DAVIS, Charles H. et KEATING, Peter, «Le Québec face aux biotechnologies», Revue Politique, Innovations et politiques technologiques, $\mathrm{n}^{\circ} 8,1985$, pp. 77-101.

CAPRILES, Oswaldo, «Le pouvoir politique et les médias en Amérique latine», Revue Politique, Les médias et les pouvoirs, $\mathrm{n}^{\circ} 2,1982$, pp. 69-95.

CARTER, Richard et BOUCHER, Michel, «Dynamique politique et déréglementation», Revue Politique, L'État privé, $\mathrm{n}^{\circ} 11$, 1987, pp. 107-125.

CARTER, Richard, «Les causes et les remèdes de la «crise des finances publiques au Québec», Revue Politique, La crise des finances publiques du Québec, n 3, 1983, pp. 91-117.

CHAMPAGNE, Maurice, «Les questions écrites dans le processus parlementaire», Revue Politique, Les médias et les pouvoirs, $\mathrm{n}^{\circ} 2,1982$, pp. 143-152. 
ClICHE, «Yvan, Lettre à Denis Monière», Revue Politique, Les intellectuels et le pouvoir, $\mathrm{n}^{\circ} 1,1982, \mathrm{pp} .89-91$.

CLOUTIER, Édouard, NADEAU, Richard et GUAY, Jean J, «Étude du mouvement de l'opinion causé par le jugement de la Cour suprême sur l'avortement», Revue Politique, Paradigmes et scientificité, $\mathrm{n}^{\circ} 15,1989, \mathrm{pp}$. 53-77.

CLOUTIER, Édouard, «Représentation politique et représentation statistique du peuple», Revue Politique, Libéralisme et démocratie, $\mathrm{n}^{\circ}$ 9, 1986, pp. 39-59.

COUTURE, Denise, «Les transformations du corps médical québécois», Revue Politique, Mouvements et acteurs, $n^{\circ} 12$, 1987, pp. 37-62.

CRETE, Jean et BLAIS, André, «La clientèle péquiste en 1985: caractéristiques et évolution», Revue Politique, Opinions et votes, $\mathrm{n}^{\circ} 10,1986, \mathrm{pp} .5-29$.

CRETE, Jean et André BLAIS «La presse et la politique municipale dans deux villes du Québec», Revue Politique, Les médias et les pouvoirs, $n^{\circ} 2,1982$, pp. 41-67.

CROZIER, Michel, «Michel Crozier et l'étude des organisations», Entrevue réalisée par Stéphane Dion, Revue Politique, Mouvements et acteurs, $\mathrm{n}^{\circ} 12,1987$, pp. 11-120.

DALPE, Robert, «Les politiques canadiennes de l'industrie aérospatiale», Revue Politique, Innovations et politiques technologiques, $\mathrm{n}^{\circ}$ 8, 1985, pp. 103-129.

DAVIS, Charles H., CAMBrosio, Alberto et KEATING, Peter, «Le Québec face aux biotechnologies», Revue Politique, Innovations et politiques technologiques, $\mathrm{n}^{\circ} 8,1985$, pp. 77-101.

DE SEVE, Micheline et LAMOUREUX, Diane, «Faut-il laisser notre sexe au vestiaire?», Revue Politique, Paradigmes et scientificité, $n^{\circ} 15,1989$, pp. 5-22.

DE SEVE, Micheline et DUGUAY, Claire, «Tant d'amarres à larguer: une analyse des pratiques du mouvement des femmes», Revue Politique, Femmes et pouvoir, $\mathrm{n}^{\circ}$ 5, 1984, pp. 51-73. 
DEBRESSON, Christian, «À l'ombre de la dynamo technologique: technologie, innovation et pôle de développement», Revue Politique, Opinions et votes, $\mathrm{n}^{\circ} 10,1986, \mathrm{pp} .55-90$.

DENIS, Roch et DENIS, Serge, «Un syndicalisme non-partisan durant les années péquistes», Revue Politique, Opinions et votes, $\mathrm{n}^{\circ} 10,1986, \mathrm{pp}$. 91-116.

DENIS, Serge et DENIS, Roch, «Un syndicalisme non-partisan durant les années péquistes», Revue Politique, Opinions et votes, $\mathrm{n}^{\circ} 10,1986, \mathrm{pp}$. 91-116.

DESOER, Frank, «Intelligentsia et médias: de l'éducation populaire au pouvoir", Revue Politique, Les médias et les pouvoirs, $\mathrm{n}^{\circ} 2,1982$, pp. 97-116.

DION, Stéphane et BLAIS, André, «Trop d'État? Un baromètre de l'opinion», Revue Politique, L'État privé, $\mathrm{n}^{\circ} 11,1987$, pp. 43-72.

DION, Stéphane, «Libéralisme et démocratie: plaidoyer pour l'idéologie dominante», Revue Politique, Libéralisme et démocratie, ${ }^{\circ} 9,1986$, pp. 5-38.

DUCATENZEILER, Graciela, «Ouverture politique, transition démocratique et classe ouvrière en Argentine», Revue Politique, Mouvements et acteurs, $\mathrm{n}^{\circ} 12,1987$, pp. 63-91.

DUCATENZEILER, Graciela, BOISMENU, Gérard et ANDERSON, Frances, "L'aide directe fédérale à l'innovation industrielle», Revue Politique, Innovations et politiques technologiques, $n^{\circ} 8,1985$, pp. 45-76.

DUGUAY, Claire et DE SEVE, Micheline, «Tant d'amarres à larguer: une analyse des pratiques du mouvement des femmes», Revue Politique, Femmes et pouvoir, $\mathrm{n}^{\circ} 5$, 1984, pp. 51-73.

ÉLIE, Bernard, «Crise de l'État ou crise du capitalisme», Revue Politique, La crise des finances publiques du Québec, $\mathrm{n}^{\circ} 3$, 1983, pp. 117-127.

FORTIN, Louise E., «La politique technologique québécoise», Revue Politique, Innovations et politiques technologiques, $n^{\circ} 8,1985$, pp. 23-44.

FOURNIER, Bernard, «Lorsque la modernité vient d'Orient», Revue Politique, Crise de décision, $\mathrm{n}^{\circ} 13,1988$, pp. 111-136. 
GABOURY, Jean-Pierre, «Pour un Parlement fédéral plus démocratique», Revue Politique, La réforme des institutions politiques, $\mathrm{n}^{\circ} 6,1984, \mathrm{pp} .127-144$.

GAY, Daniel, «La présence du Québec en Amérique latine», Revue Politique, Projection internationale du Québec, $\mathrm{n}^{\circ} 7,1985$, pp. 33-52.

GERVAIS, Myriam, «La politique africaine du Québec de 1960 à 1984», Revue Politique, Projection internationale du Québec, $\mathrm{n}^{\circ}$ 7, 1985, pp. 53-66.

GIROUX, France, «Les incohérences d'un libéralisme exacerbé», Revue Politique, Libéralisme et démocratie, $\mathrm{n}^{\circ} 9,1986$, pp. 99-110.

GOW, James Iain, «Repenser l'État et son administration», Revue Politique, L'État privé, $\mathrm{n}^{\circ} 11,1987$, pp. 5-41.

Gow, James Iain, «La réforme institutionnelle de la fonction publique de 1983», Revue Politique, La réforme des institutions politiques, $n^{\circ} 6,1984$, pp. 51-101.

GOW, James Iain, «Perspectives historiques sur les compressions budgétaires», Revue Politique, La crise des finances publiques du Québec, n 3, 1983, pp. 55-25.

GUAY, Jean J., NADEAU, Richard et CLOUTIER, Édouard, «Étude du mouvement de l'opinion causé par le jugement de la Cour suprême sur l'avortement», Revue Politique, Paradigmes et scientificité, $\mathrm{n}^{\circ} 15,1989$, pp. 53-77.

HARVEY, Jean, «L'investissement politique du corps et l'Étatprovidence canadien», Revue Politique, Sport et politique et $L e N P D, \mathrm{n}^{\circ} 14,1988$, pp. 5-35.

HOULE, François, «Stratégie économique et restructuration de l'État au Canada», Revue Politique, La crise des finances publiques du Québec, $\mathrm{n}^{\circ} 3,1983$, pp. 63-87.

HUDON, Raymond, «La commission Macdonald: principes et préceptes», Revue Politique, Libéralisme et démocratie, $n^{\circ} 9,1986$, pp. 111-145.

HUDON, Raymond, «La construction de solidarités nationales contre le mouvement syndical», Revue Politique, Crise et changements idéologiques, $\mathrm{n}^{\circ} 4,1983$, pp. 129-163. 
KeatiNG, Peter, DAVIS, Charles H. et CAMBRosio, Alberto, «Le Québec face aux biotechnologies», Revue Politique, Innovations et politiques technologiques, $n^{\circ} 8,1985, \mathrm{pp}$. 77-101.

KRIEBER, Janine, «La démocratie du secret: le contrôle des activités de renseignements au Canada», Revue Politique, Crise de décision, $\mathrm{n}^{\circ} 13,1988$, pp. 37-62.

LACHAPELLE, Guy, «Les répondants discrets et l'élection québécoise de 1985», Revue Politique, Opinions et votes, no. 10,1986, pp. $31-54$.

LAFOREST, Guy, «Démocratie et libéralisme: pour une approche historico-théorique», Revue Politique, Crise de décision, $n^{\circ} 13,1988$, pp. 87-109.

LAFOREST, Guy, «Penser l'État: Gérard Bergeron, un Aufklarer québécois», Revue Politique, Libéralisme et démocratie, $\mathrm{n}^{\circ}$ 9, 1986, pp. 147-166.

LAMONT, Michèle, «Les rapports politiques au sein du mouvement des femmes au Québec», Revue Politique, Femmes et pouvoir, $\mathrm{n}^{\circ}$ 5, 1984, pp. 75-107.

LAMONT, Michèle, «Le pouvoir des intellectuels», Revue Politique, Les intellectuels et le pouvoir, $\mathrm{n}^{\circ} 1,1982, \mathrm{pp}$. 19-46.

LAMOUREUX, André, «L'élection fédérale de 1988 et la défaillance du NPD», Revue Politique, Paradigmes et scientificité, $\mathrm{n}^{\circ}$ 15, 1989, pp. 79-103.

LAMOUREUX, André, «Le NPD de 1984 à 1988; à la recherche d'un nouvel élan», Revue Politique, Sport et politique et Le NPD, $\mathrm{n}^{\circ} 14,1988$, pp. 83-118.

LAMOUREUX, Diane et DE SEVE, Micheline, «Faut-il laisser notre sexe au vestiaire?», Revue Politique, Paradigmes et scientificité, $\mathrm{n}^{\circ} 15,1989, \mathrm{pp} .5-22$.

LANDRY, Réjean, «L'orientation interventionniste des idéologies des partis politiques québécois depuis 1970», Revue Politique, Crise de décision, $\mathrm{n}^{\circ} 13,1988$, pp. 5-32.

LANDRY, Réjean, «La nouvelle analyse institutionnelle», Revue Politique, La réforme des institutions politiques, $n^{\circ} 6$, 1984, pp. 63-85. 
LANGLOIS, Simon et SAUVAGEAU, Florian, «Les journalistes des quotidiens québécois et leur métier», Revue Politique, Les médias et les pouvoirs, $\mathrm{n}^{\circ} 2,1982, \mathrm{pp}$. 5-39.

LAROCHELLE, Charles, «La France entre le socialisme et l'austérité», Revue Politique, Crise et changements idéologiques, $\mathrm{n}^{\circ} 4,1983$, pp. 105-127.

LATOUCHE, Daniel, «Une prospective des relations France-Québec», Revue Politique, Projection internationale du Québec, $\mathrm{n}^{\circ} 7,1985$, pp. 67-86.

LAURIN, Pierre, «Une relecture de J.F. Kennedy», Revue Poliique, Les intellectuels et le pouvoir, $\mathrm{n}^{\circ} 1,1982, \mathrm{pp} .73-88$.

LAVOIE, Marie et LEMIEUX, Vincent, «La réforme du système électoral», Revue Politique, La réforme des institutions politiques, $\mathrm{n}^{\circ} 6,1984$, pp. 33-50.

LECLERC, Michel, «La notion de discipline scientifique», Revue Politique, Paradigmes et scientificité, $\mathrm{n}^{\circ} 15,1989$, pp. 2351.

LEMIEUX, Vincent, «Le pouvoir prédominant dans les statuts des partis: le cas du NPD», Revue Politique, Sport et politique et $L e N P D, \mathrm{n}^{\circ} 14,1988, \mathrm{pp} .63-82$.

LEMIEUX, Vincent et LAVOIE, Marie, «La réforme du système électoral», Revue Politique, La réforme des institutions politiques, $\mathrm{n}^{\circ} 6,1984$, pp. 33-50.

LIPIETZ, Alain, «Crise de l'État-providence: idéologies, réalités et enjeux dans la France des années 1980», Revue Politique, Crise et changements idéologiques, $\mathrm{n}^{\circ} 4,1983$, pp. 55-103.

MAHEU, Louis, «Crise sociale, mouvements sociaux et pratiques du changement social», Revue Politique, Crise et changements idéologiques, $\mathrm{n}^{\circ} 4$, 1983, pp. 27-54.

MAROIS, Michel, «Sport, violence et politique; prologue à une interprétation politique du sport et de la violence des foules sportives", Revue Politique, Sport et politique et Le NPD, $\mathrm{n}^{\circ} 14,1988$, pp. 37-61.

Mc ROBERTS, Kenneth et BLAIS, André, «Dynamique et contraintes des finances publiques au Québec», Revue Politique, La crise des finances publiques du Québec, $\mathrm{n}^{\circ} 3$, 1983, pp. 27-62. 
MERCIER, Jean et PARENT, Richard, "Conflit entre marché et État dans la société technicienne», Revue Politique, Innovations et politiques technologiques, $\mathrm{n}^{\circ} 8,1985$, pp. 5-21.

NADEAU, Richard, GUAY, Jean J. et CLOUTIER, Édouard, «Étude du mouvement de l'opinion causé par le jugement de la Cour suprême sur l'avortement», Revue Politique, Paradigmes et scientificité, $\mathrm{n}^{\circ} 15,1989$, pp. 53-77.

ORBAN, Edmond, «Les scandales politiques aux États-Unis», Revue Politique, Mouvements et acteurs, $\mathrm{n}^{\circ} 12,1987, \mathrm{pp}$. 93-110.

PARENT, Richard et MERCIER, Jean, «Conflit entre marché et État dans la société technicienne», Revue Politique, Innovations et politiques technologiques, $\mathrm{n}^{\circ} 8,1985, \mathrm{pp} .5-21$.

PARE, Jean, «Que doit faire l'État contre les monopoles de presse?», Revue Politique, Les médias et les pouvoirs, no. 2,1982 , pp. 117-133.

PELLETIER, Réjean, «Les fonctions du député: bilan des réformes parlementaires à Québec», Revue Politique, La réforme des institutions politiques, $n^{\circ} 6,1984$, pp. 145-154.

PERRON, Bruno, «Les contraintes dans les relations entre le Québec et les États-Unis», Revue Politique, Projection internationale du Québec, $\mathrm{n}^{\circ}$ 7, 1985, pp. 9-31.

PICARD, Jean-Claude, «Que doit faire l'État contre les monopoles de presse?», Revue Politique, Les médias et les pouvoirs, $n^{\circ} 2,1982$, pp. 133-142.

QUESNEL, Louise, «La démocratie municipale au Québec», Revue Politique, Libéralisme et démocratie, $\mathrm{n}^{\circ} 9,1986, \mathrm{pp} .61-$ 97.

RIVET, Jacques, «L'interrogatoire public», Revue Politique, Les intellectuels et le pouvoir, $\mathrm{n}^{\circ} 1,1982$, pp. 63-92.

ROCHER, François, «La bourgeoisie canadienne et les paramètres de l'alliance continentale», Revue Politique, Sport et politique et Le NPD, $\mathrm{n}^{\circ} 14,1988$, pp. 119-155.

SAUVAGEAU, Florian et LANGLOIS, Simon, «Les journalistes des quotidiens québécois et leur métier», Revue Politique, Les médias et les pouvoirs, $\mathrm{n}^{\circ} 2,1982, \mathrm{pp} .5-39$. 
SEYMOUR, Martin Lipset, «Les transformations idéologiques aux États-Unis», entrevue réalisée par Michèle Lamont, Revue Politique, Crise et changements idéologiques, $n^{\circ} 4,1983$, pp. 5-26.

SIMARD, Carolle, «La recherche en administration publique au Québec: 1960-1980», Revue Politique, L'État privé, $\mathrm{n}^{\circ} 11$, 1987, pp. 73-106.

SIMARD, Carolle, «Changement et insertion des femmes dans le système politique», Revue Politique, Femmes et pouvoir, $n^{\circ} 5,1984$, pp. $27-49$.

THÉRIAULT, J. Yvon, «Mouvements sociaux et nouvelle culture politique», Revue Politique, Mouvements et acteurs, $\mathrm{n}^{\circ} 12$, 1987, pp. 5-36.

TOURAINE, Alain, «L'itinéraire d'Alain Touraine», Entrevue réalisée par Philippe Faucher, Revue Politique, Projection internationale du Québec, $\mathrm{n}^{\circ}$ 7, 1985, pp. 87-97. 


\section{Index des articles publiés dans la revue Politique nos 1-15 (par numéro)}

REVUE POLITIQUE, Les intellectuels et le pouvoir, $\mathrm{n}^{\circ} 1,1982$.

BERGERON, Gérard, «De la tour d'ivoire à la place publique».

LAMONT, Michèle, "Le pouvoir des intellectuels».

BERNARD, André, «Les «experts» et le pouvoir».

RIVET, Jacques, «L'interrogatoire public».

LAURIN, Pierre, «Une relecture de J.F. Kennedy»

CLICHE, Yvan, «Lettre à Denis Monière».

REVUE POLITIQUE, Les médias et les pouvoirs, $\mathrm{n}^{\circ} 2,1982$.

LANGLOIS, Simon et SAUVAGEAU, Florian, «Les journalistes des quotidiens québécois et leur métier».

BLAIS, André et CRETE, Jean, «La presse et la politique municipale dans deux villes du Québec».

CAPRILES, Oswaldo, «Le pouvoir politique et les médias en Amérique latine».

DESOER, Frank, «Intelligentsia et médias: de l'éducation populaire au pouvoir». 
PARÉ, Jean, «Que doit faire l'État contre les monopoles de presse?».

PICARD, Jean-Claude, «Que doit faire l'État contre les monopoles de presse?».

CHAMPAGNE, Maurice, «Les questions écrites dans le processus parlementaire».

REVUE POLITIQUE, La crise des finances publiques du Québec, $\mathrm{n}^{\circ} 3,1983$.

Gow, James Iain, «Perspectives historiques sur les compressions budgétaires».

BLAIS, André et Mc ROBERTS, Kenneth, «Dynamique et contraintes des finances publiques au Québec».

HOULE, François, «Stratégie Économique et restructuration de l'État au Canada».

CARTER, Richard, «Les causes et les remèdes de la «crise des finances publiques au Québec».

ÉLIE, Bernard, «Crise de l'État ou crise du capitalisme».

REVUE POLITIQUE, Crise et changements idéologiques, $\mathrm{n}^{\circ} 4$, 1983.

SEYMOUR, Martin Lipset, «Les transformations idéologiques aux États-Unis», entrevue réalisée par Michèle Lamont.

MAHEU, Louis, «Crise sociale, mouvements sociaux et pratiques du changement social».

LIPIETZ, Alain, «Crise de l'État-providence: idéologies, réalités et enjeux dans la France des années 1980».

LAROCHELLE, Charles, «La France entre le socialisme et l'austérité».

HUDON, Raymond, «La construction de solidarités nationales contre le mouvement syndical». 
REVUE POLITIQUE, Femmes et pouvoir, $\mathrm{n}^{\circ}$ 5, 1984.

BOILY, Nicole, «Les femmes en politique, encore une exception», entrevue réalisée par Carolle Simard et Denis Monière.

SIMARD, Carolle, «Changement et insertion des femmes dans le système politique».

DUGUAY, Claire et DE SEVE, Micheline, «Tant d'amarres à larguer: une analyse des pratiques du mouvement des femmes».

LAMONT, Michèle, «Les rapports politiques au sein du mouvement des femmes au Québec».

ANDREW, Caroline, «Les femmes et la consommation collective: les enjeux de l'engagement politique».

REVUE POLITIQUE, La réforme des institutions politiques, $\mathrm{n}^{\circ} 6$, 1984.

LANDRY, Réjean, «La nouvelle analyse institutionnelle».

LEMIEUX, Vincent et LAVOIE, Marie, «La réforme du système électoral».

GoW, James Iain, «La réforme institutionnelle de la fonction publique de 1983».

BOUCHER, Michel, «Le référendum de mai 1980: présentation de quelques résultats statistiques».

GABOURY, Jean-Pierre, «Pour un Parlement fédéral plus démocratique».

PELLETIER, Réjean, «Les fonctions du député: bilan des réformes parlementaires à Québec».

REVUE POLITIQUE, Projection internationale du Québec, $\mathrm{n}^{\circ} 7$, 1985.

PERRON, Bruno, «Les contraintes dans les relations entre le Québec et les États-Unis».

GAY, Daniel, «La présence du Québec en Amérique latine».

GERVAIS, Myriam, «La politique africaine du Québec de 1960 à 1984». 
LATOUCHE, Daniel, «Une prospective des relations FranceQuébec".

TOURAINE, Alain, «L'itinéraire d'Alain Touraine», Entrevue réalisée par Philippe Faucher.

REVUE POLITIQUE, Innovations et politiques technologiques, $\mathrm{n}^{\circ} 8,1985$.

MERCIER, Jean et PARENT, Richard, «Conflit entre marché et État dans la société technicienne».

FORTIN, Louise E., «La politique technologique québécoise». BOISMENU, Gérard, DUCATENZEILER, Graciela et ANDERSON, Frances, «L'aide directe fédérale à l'innovation industrielle».

CAMBROSIO, Alberto, DAVIS, Charles H. et KEATING, Peter, "Le Québec face aux biotechnologies».

DALPE, Robert, «Les politiques canadiennes de l'industrie aérospatiale».

REVUE POLTTQUE, Libéralisme et démocratie, $\mathrm{n}^{\circ}$ 9, 1986.

DION, Stéphane, «Libéralisme et démocratie: plaidoyer pour l'idéologie dominante».

CLOUTIER, Édouard, «Représentation politique et représentation statistique du peuple».

QUESNEL, Louise, «La démocratie municipale au Québec».

GIROUX, France, «Les incohérences d'un libéralisme exacerbé».

HUDON, Raymond, «La commission Macdonald: principes et préceptes».

LAFOREST, Guy, «Penser l'État: Gérard Bergeron, un Aufklarer québécois».

REVUE POLITIQUE, Opinions et votes, $\mathrm{n}^{\circ} 10,1986$.

BLAIS, André et CRETE, Jean, «La clientèle péquiste en 1985: caractéristiques et évolution».

LACHAPELLE, Guy, «Les répondants discrets et l'élection québécoise de 1985». 
DEBRESSON, Christian, «À l'ombre de la dynamo technologique: technologie, innovation et pôle de développement».

DENIS, Roch et DENIS, Serge, «Un syndicalisme non-partisan durant les années péquistes».

REVUE POLITIQUE, L'État privé, $\mathrm{n}^{\circ} 11,1987$.

GOW, James Iain, «Repenser l'État et son administration».

BLAIS, André et DION, Stéphane, «Trop d'État? Un baromètre de l'opinion».

SIMARD, Carolle, «La recherche en administration publique au Québec: 1960-1980».

CARTER, Richard et BOUCHER, Michel, «Dynamique politique et déréglementation».

REVUE POLITIQUE, Mouvements et acteurs, n 12, 1987.

THÉRIAULT, J. Yvon, «Mouvements sociaux et nouvelle culture politique».

COUTURE, Denise, «Les transformations du corps médical québécois».

DUCATENZEILER, Graciela, «Ouverture politique, transition démocratique et classe ouvrière en Argentine».

ORBAN, Edmond, «Les scandales politiques aux États-Unis».

CROZIER, Michel, «Michel Crozier et l'étude des organisations», Entrevue réalisée par Stéphane Dion.

REVUE POLITIQUE, Crise de décision, nº 13, 1988.

BAUER, Julien, «Résolution des conflits et crise de décision».

KRIEBER, Janine, «La démocratie du secret: le contrôle des activités de renseignements au Canada».

LANDRY, Réjean, «L'orientation interventionniste des idéologies des partis politiques québécois depuis 1970».

LAFOREST, Guy, «Démocratie et libéralisme: pour une approche historico-théorique».

FOURNIER, Bemard, «Lorsque la modernité vient d'Orient». 
REVUE POLITIQUE, Sport et politique et Le NPD, $\mathrm{n}^{\circ} 14,1988$.

HARVEY, Jean, «L'investissement politique du corps et l'Étatprovidence canadien».

MAROIS, Michel, «Sport, violence et politique; prologue à une interprétation politique du sport et de la violence des foules sportives».

LEMIEUX, Vincent, «Le pouvoir prédominant dans les statuts des partis: le cas du NPD».

LAMOUREUX, André, «Le NPD de 1984 à 1988; à la recherche d'un nouvel élan».

ROCHER, François, «La bourgeoisie canadienne et les paramètres de l'alliance continentale».

REVUE POLITIQUE, Paradigmes et scientificité, nº 15, 1989.

LAMOUREUX, Diane et DE SEVE, Micheline, «Faut-il laisser notre sexe au vestiaire?»

LECLERC, Michel, «La notion de discipline scientifique».

GUAY, Jean J., NADEAU, Richard et CLOUTIER, Édouard, «Étude du mouvement de l'opinion causé par le jugement de la Cour suprême sur l'avortement».

LAMOUREUX, André, «L'élection fédérale de 1988 et la défaillance du NPD». 